\title{
Reemergence of Syphilitic Uveitis Masquerading as Other Diseases: A Report of Two Cases
}

\author{
Danny S. Ng ${ }^{\mathrm{a}}$ Ian Y. Wong ${ }^{\mathrm{b}}$ Clement W. Chan \\ aDepartment of Ophthalmology, Tung Wah Eastern Hospital, and ${ }^{b}$ Eye Institute, \\ Queen Mary Hospital, University of Hong Kong, Hong Kong, SAR, China
}

\section{Key Words}

Neurosyphilis · Uveitis · Harada's disease · Behçet's disease

\begin{abstract}
During a 6-month period in 2010, 2 patients with uveitis were examined at our department and diagnosed with ocular syphilis. They initially presented with symptoms and signs resembling Harada's disease and Behçet's disease and were therefore treated with systemic steroids with suboptimal responses. When laboratory workup revealed neurosyphilis, they were given a course of intravenous penicillin $G$, which led to significant clinical and visual improvement. Epidemiological data indicates a worldwide reemergence of syphilis and a high degree of suspicion is necessary in view of its multitude of presenting ocular signs without pathognomonic features.
\end{abstract}

\section{Introduction}

Although syphilis seemed to be under control after the advent of antibiotics, recent epidemiological data showed drastically increased incidence in Europe and the United States since the late 1990s and 2000s [1,2]. Worldwide, there are an estimated 12 million new cases of syphilis every year, over $90 \%$ of which occur in developing countries [3]. The Hong Kong Social Hygiene Service reported around 1,400 new cases of syphilis per year in the 1970s followed by a decline to about 300 new cases per year in the early 1990s [4]. Since 1998, the number gradually increased to over 1,000 new cases of syphilis up till 2010 [4].

Ocular syphilis is an uncommon but diagnostically important manifestation of the disease. It was reported to occur in 2.5 to $5 \%$ of patients with tertiary syphilis [5]. A multitude of presenting ocular signs has been described among both human immunodeficiency virus (HIV)-positive and HIV-negative patients. It may be the initial presentation in the eye, occurring in one or both eyes, without obvious systemic 
manifestations. The diagnosis of ocular syphilis is challenging because of its protean features and lack of distinguishing characteristics.

During a 6-month period in 2010, 2 patients with widely disparate presenting ocular symptoms and signs were examined at our department and diagnosed with ocular syphilis. We reported the clinical findings and management of these 2 cases which illustrate the importance of a high degree of suspicion, prompt diagnosis and appropriate treatment of ocular syphilis in light of its reemergence in Hong Kong.

\section{Case Reports}

\section{Case 1}

A 67-year-old heterosexual man presented with a 1-week history of decreased vision in both eyes. On examination, Snellen visual acuity was $6 / 60$ and $6 / 18$ in his right and left eye, respectively. A relative afferent papillary defect in the right eye was noted. Slit-lamp examination was unremarkable with absence of cells in the anterior chamber and anterior vitreous. Neither were there any keratic precipitates, iris nodules or posterior synechiae. On dilated fundus examination, both optic discs were hyperemic with exudative retinal detachment in the macula (fig. 1 a b). There were no retinal exudates, hemorrhages, or signs of vasculitis, and the vitreous was clear. Systemic review elicited no significant finding either.

Two days later, the patient reported a further decrease in vision bilaterally. Visual acuity was hand movement in his right eye and count fingers at 1 foot in his left eye. Intravenous fluorescein angiography showed leakage from the retinal pigment epithelial layer and optic discs bilaterally (fig. 1c and d). Complete blood count and immune markers were unremarkable except for a raised erythrocyte sedimentation rate (ESR) of $36 \mathrm{~mm} / \mathrm{h}$.

Given the acute onset of vision loss secondary to exudative retinal detachment, the hyperemic optic disc, which showed leakage on fluorescein angiograph, together with an absence of ocular trauma, the provisional diagnosis of Harada's disease (uveitic phase) was made. The patient was treated with topical and oral prednisolone $1 \mathrm{mg} / \mathrm{kg} /$ day and the visual acuity improved to $6 / 30$ and 6/12 in his right and left eye, respectively. However, 5 weeks into steroid treatment, anterior chamber cells started to appear in his right eye. Indirect ophthalmoscopy revealed creamy-yellow retinal infiltrates along the inferotemporal arcade, hemorrhage and associated shallow subretinal exudate in the right eye (fig. 2a, b). The initial finding of exudative retinal detachment in the macula had resolved but disc hyperemia was still present in both eyes. On fluorescein angiography there was early hyperfluorescence and late leakage associated with the inferotemporal lesion in the right eye (fig. 2c) and bilateral disc. With the history of immunosuppression with oral steroid, the diagnosis of acute retinal necrosis was made and the patient was treated with a course of intravenous aciclovir and systemic prednisolone was gradually tapered. One week after therapy was started, the patient complained of a headache and systemic laboratory workup revealed a positive Venereal Disease Research Laboratory (VDRL) test and subsequent fluorescent treponemal antibody absorption (FTA-Abs) test was reactive. Cerebrospinal fluid analysis demonstrated 160 leukocytes per microliter, elevated protein and a positive VDRL test. The patient was treated for neurosyphilis with a 14-day course of intravenous penicillin (4 million units every $4 \mathrm{~h}$ ).

Over the next month, the patient's visual acuity improved to $6 / 12$ in the right eye and to $6 / 9$ in the left eye. Right-eye iridocyclitis, subretinal fluid and infiltrates together with optic disc hyperemia resolved completely.

\section{Case 2}

A 30-year-old heterosexual man with good past health presented with a 2-week history of decreased vision in both eyes. He also complained of oral and genital ulcers, and bilateral maculopapular rash in 
the palms and forearms. He initially presented to a private ophthalmologist, who found bilateral vitritis and made the provisional diagnosis of Behçet's disease. Oral prednisolone $60 \mathrm{mg}$ daily was given for 4 days prior to referral to our center for further management. Snellen visual acuity was $6 / 12$ in the right eye and 6/18 in the left eye. Slit-lamp examination revealed quiet anterior chamber. Fundoscopic examination showed 1+ anterior vitreous cells, disc hyperemia and vitritis bilaterally. Laboratory workup was positive for VDRL and FTA-Abs tests and showed a raised ESR of $102 \mathrm{~mm} / \mathrm{h}$. HIV test was positive. Infectious disease consultation was obtained and lumbar puncture was performed.

Cerebrospinal fluid analysis demonstrated 133 leukocytes per microliter, elevated protein and a positive VDRL test. When the diagnosis of neurosyphilis was conveyed to the patient, he revealed a history of unprotected sexual exposure. The patient received a 14-day course of intravenous penicillin (4 million units every $4 \mathrm{~h}$ ) and oral steroid was tapered. His Snellen visual acuity had improved to 6/9 in both eyes upon discharge.

\section{Discussion}

The diagnosis of ocular syphilis is often elusive because of the protean features and lack of distinguishing clinical characteristics. Because of its ubiquitous nature, it has been coined as 'the great imitator'. In both cases reported, the diagnosis of ocular syphilis was not considered prior to laboratory tests and provisional diagnoses of Harada's disease and Behçet's disease were made.

The most common presentation of ocular syphilis, reported by Anshu et al. [6] in a series of 22 consecutive patients in Singapore, was nongranulomatous anterior uveitis $(81.8 \%)$, followed by vitritis (65.4\%), papillitis $(27.5 \%)$, scleritis/episcleritis $(22.7 \%)$, interstitial keratitis (22.7\%), granulomatous uveitis (13.7\%), vasculitis $(13.7 \%)$ and chorioretinitis (13.7\%). Exudative retinal detachment has rarely been reported as the initial consequence of ocular syphilis. Jumper et al. [7] reported a series of 3 cases with exudative retinal detachment but all were presented together with focal retinitis. However, case 1 from our report presented with bilateral exudative retinal detachment without focal retinitis initially. Provisional diagnosis of Harada's disease led to the use of corticosteroids which may have caused the subsequent manifestation of focal retinitis 5 weeks after commencement of the steroids.

Fu et al. [8] described a multicenter series of 8 patients who had superficial creamyyellow retinal precipitates with syphilitic retinitis regardless of sexual preference or HIV status [8]. In our first case, we also found such superficial retinal precipitates with similar optical coherence tomography (OCT) appearance (fig. 2d) as in a report by Reddy et al. [9]. All of the reported cases had rapid resolution of these precipitates after treatment with penicillin G. It was hypothesized that these were pre-retinal collections of leukocytes, representing an exaggerated ocular response to syphilitic infection as a result of immune reconstitution [8]. This distinctive feature may help differentiate ocular syphilis from other etiologies of retinitis such as herpes and cytomegalovirus infection.

Ophthalmic manifestations have been reported in all stages of syphilis. Nonetheless, the presence of optic neuritis and retinitis are generally considered as neurosyphilis and should be managed accordingly. The United States Center for Disease Control (CDC) Sexually Transmitted Diseases Treatment Guidelines recommended treatment of neurosyphilis with penicillin G (18-24 million units daily) for 10 to 14 days [8]. Increased leukocyte counts and positive VDRL test in cerebrospinal fluid analysis was highly specific but not sensitive [10]. Hence, a negative result should not eliminate the suspicion of neurosyphilis. In addition, the VDRL titer may not be proportional to the level of 
disease activity, rendering it ineffective for monitoring the effects of treatment. If pleocytosis in the cerebrospinal fluid was observed at presentation, monitoring was suggested at 6-month intervals until the cell count normalized [10]. The use of systemic corticosteroids as an adjunct for posterior uveitis, scleritis and optic neuritis associated with syphilis has been described [3]. In addition, it may prevent the Jarisch-Herxheimer reaction resulting from a hypersensitivity reaction to treponemal antigens that are released in large numbers as spirochetes are killed [11]. HIV co-infection was found to be common (33\%) and the CDC recommended that all patients with neurosyphilis be tested for HIV [1].

Syphilis is a rare cause of uveitis, accounting for $1-5 \%$ of cases reported $[12,13]$. Diagnosing ocular syphilis was difficult because a multitude of presenting signs has been described without pathognomonic features. Patients may deny having a history of venereal disease exposure despite direct questioning. Awareness of the reemergence of syphilis reported in many parts of the world in the past decade as well as a high degree of clinical suspicion can allow ophthalmologists to diagnose and treat the disease early, having a reasonably good visual prognosis following treatment with antibiotics. 


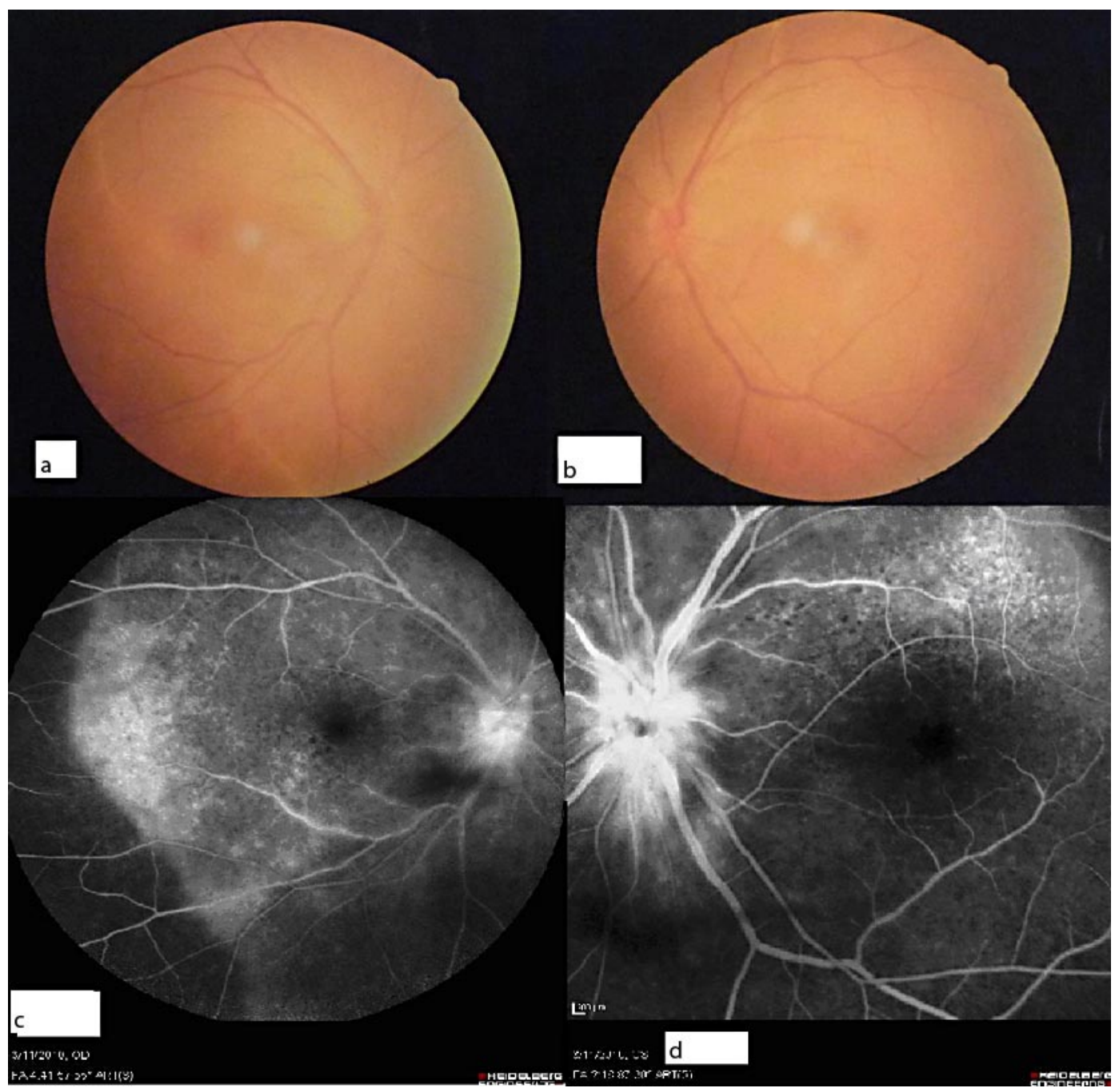

Fig. 1. Case 1. Fundus photographs of the right (a) and left (b) eyes reveal disc hyperemia in both eyes. Exudative retinal detachment was present in both eyes. Fluorescein angiography of the right (c) and left (d) eyes revealed disc leakage and multifocal subretinal leakage. 


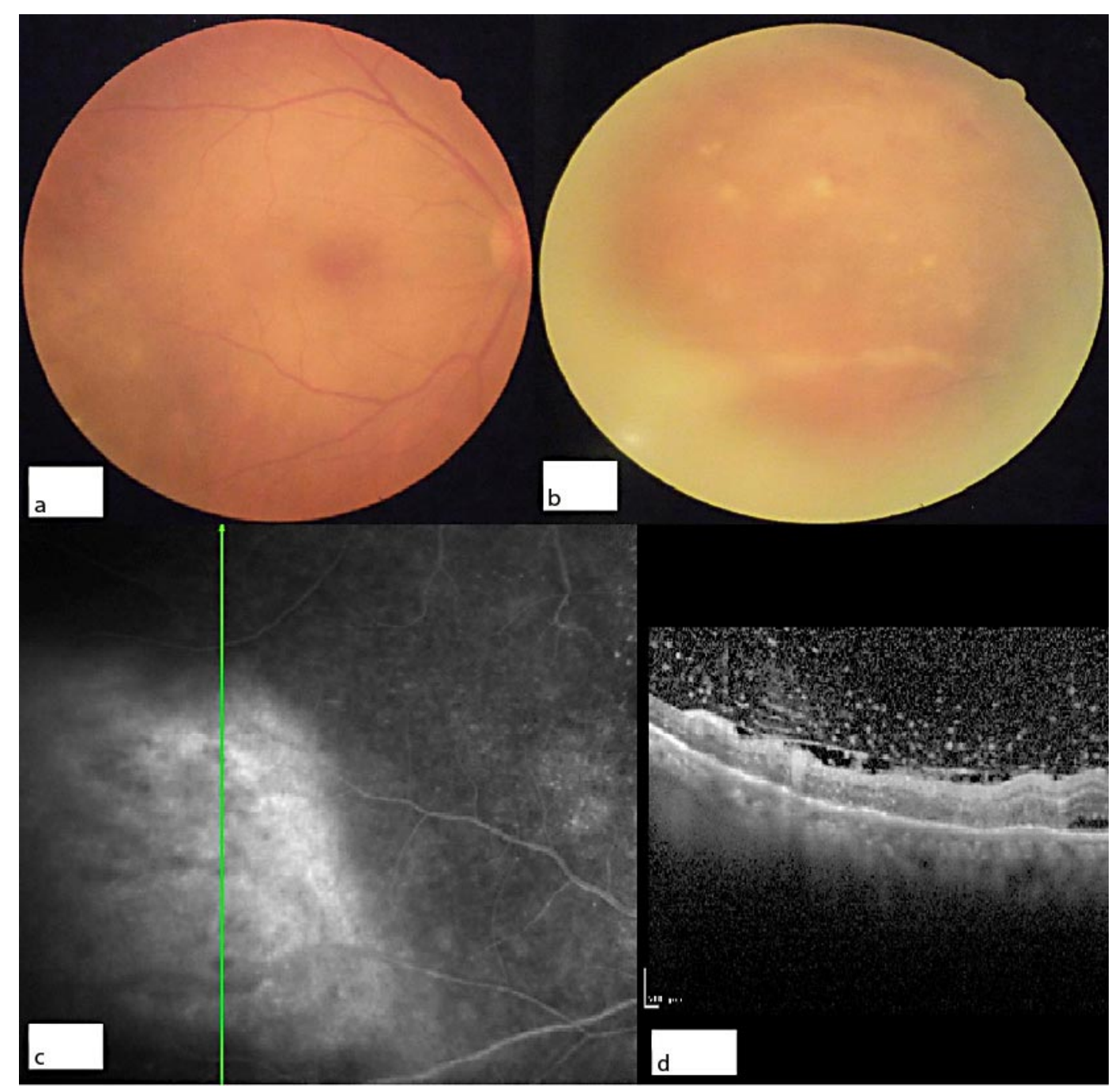

Fig. 2. Case 1. Fundus photographs of the right eye with focal retinitis near the inferotemporal arcade (a). Magnified view of the lesion (b) revealed creamy-yellow superficial precipitates, hemorrhage and shallow subretinal exudate. Fluorescein angiography of the right eye (c) revealed leakage and subretinal pooling at the inferotemporal arcade corresponding to the site of focal retinitis and disc leakage. Spectralis optical coherence tomography (d) found pre-retinal signals at the boundary of detached vitreous corresponding to the creamy-yellow superficial precipitates.

\section{References}

1 Doris JP, Saha K, Jones, NP, Sukthankar A: Ocular syphilis: the new epidemic. Eye (Lond) 2006;20:703-705.

2 Chao JR, Khurana RN, Fawzi AA, et al: Syphilis: reemergence of an old adversary. Ophthalmology 2006;113:2074-2079.

-3 Hook EW 3rd, Peeling RW: Syphilis control - a continuing challenge. N Engl J Med 2004;351:122-124.

4 Centre for Health Protection: Reported sexually transmitted infections (STIs) diagnosed in Social Hygiene Service. Hong Kong STD/AIDS Update. Vol 16, No. 3, Quarter 3, 2010; Available from: http://www.info.gov.hk/aids/archives/backissuestd/std10q3.pdf.

5 Aldave AJ, King JA, Cunningham ET Jr: Ocular syphilis. Curr Opin Ophthalmol 2001;12:433-441. 
6 Anshu A, Cheng CL, Chee SP: Syphilitic uveitis: an Asian perspective. Br J Ophthalmol 2008;92:594-597.

7 Jumper JM, Machemer R, Gallemore RP, Jaffe GJ: Exudative retinal detachment and retinitis associated with acquired syphilitic uveitis. Retina 2000;20:190-194.

$\checkmark 8$ Fu EX, Geraets RL, Dodds EM, et al: Superficial retinal precipitates in patients with syphilitic retinitis. Retina 2010;30:1135-1143.

-9 Reddy S, Cunningham ET Jr, Spaide RF: Syphilitic retinitis with focal inflammatory accumulations. Ophthalmic Surg Lasers Imaging 2006;37:429-431.

10 Centers for Disease Control and Prevention: Sexually transmitted diseases treatment guidelines 2002. MMWR Recomm Rep 2002;51:1-78.

11 Danesh-Meyer H, Kubis KC, Sergott RC: Not so slowly progressive visual loss. Surv Ophthalmol 1999;44:247252 .

12 Puech C, et al: Ocular manifestations of syphilis: recent cases over a 2.5-year period. Graefes Arch Clin Exp Ophthalmol 2010;248:1623-1629.

13 Barile GR, Flynn TE: Syphilis exposure in patients with uveitis. Ophthalmology 1997;104:1605-1609. 